\title{
Adiabatic Higher Order Mode Guidance in Optical Microfibres
}

\author{
Y. Jung, ${ }^{(1),{ }^{*}}$ K. Harrington, (2) S. Yerolatsitis, ${ }^{(2)}$ S. U. Alam, ${ }^{(1)}$ D. J. Richardson, ${ }^{(1)}$ and T. A. Birks ${ }^{(2)}$ \\ (1) Optoelectronics Research Centre, University of Southampton, Southampton, UK \\ (2) Department of Physics, University of Bath, Claverton Down, Bath BA2 7AY, UK \\ "Corresponding Authors: ymi@orc.soton.ac.uk
}

\begin{abstract}
Optical fibres with a logarithmic index profile can provide invariant mode field diameters along the fibre taper, which enables adiabatic mode transitions for higher-order mode microfibres. A $2 \mu m-O D$ microfibre is fabricated with an insertion loss lower than $0.11 \mathrm{~dB}$ for the $L P_{11}$ mode.
\end{abstract}

\section{Introduction}

Optical microfibers or nanofibers (having outer diameters ranging from $\sim 100 \mathrm{~nm}$ to a few $\mu \mathrm{m}$ ) [1, 2] have attracted considerable attention in the fields of optical communications, optical sensors, nonlinear optics and quantum/atom optics due to their strong evanescent light field, wavelengthscale mode profiles and large waveguide dispersion. The majority of previous studies have been conducted with single mode microfibers supporting only the fundamental mode but higher-order mode (HOM) microfibers have recently gained increasing interest for use in particle propulsion and micro-resonators [3]. For example, HOM microfibres can provide greater evanescent field extension around the taper waist compared to the fundamental mode and faster particle propulsion speeds have been observed [3]. In general, however, meeting the adiabatic taper criterion for HOMs in common optical fibres (e.g. step-index or graded index fibres) is more challenging than for the fundamental mode because the MFD changes rapidly when the mode is cutoff from the core. Consequently it is hard to achieve low loss HOM microfibres. Reduced cladding fibres (e.g. $80 \mu \mathrm{m}$ OD fibres) and/or high numerical aperture fibres have been proposed to ease the adiabatic criterion for HOMs but this still dictates the need for well controlled small taper angles ( few mrad) to realize low-loss HOM microfibres [4]. Very recently, the new concept of an optical fibre having a logarithmic (LOG) refractive index profile [5] has been proposed as a means to provide an endlessly adiabatic taper transition due to the invariant mode field diameter (MFD) under tapering. A short low-loss taper ( 2 $\mathrm{mm})$ for the fundamental mode has been experimentally demonstrated validating the approach. Importantly, these LOG fibres can support several spatial modes and all the guided modes are expected to have constant MFDs that are independent of the scale of the fibre, just as for the fundamental mode.

In this paper, we will focus our attention on HOM microfibres and investigate the advantages provided by LOG fibres for low-loss HOM microfibre fabrication.

\section{LOG fibre characterization}

Figure $1(\mathrm{a})$ shows the measured refractive index profile (RIP) of the LOG fibre [5], which is defined by $n^{2}(r)=n_{0}^{2}-N A^{2} \ln (r / \rho)$. From numerical simulation of the guided mode properties, the current LOG fibre can be used for effective two mode operation supporting $L P_{01}$ and $L P_{11}$ modes at $1550 \mathrm{~nm}$. In order to characterize experimentally the modal properties of the LOG fibre, the multimode impulse response of the fibre was first measured by selective modal excitation at the input and subsequent time-of-flight measurement at the output. As shown in Fig. 1(b), two distinguishable temporal peaks, corresponding to $\mathrm{LP}_{01}$ and $\mathrm{LP}_{11}$ modes, are clearly observed and the differential group delay

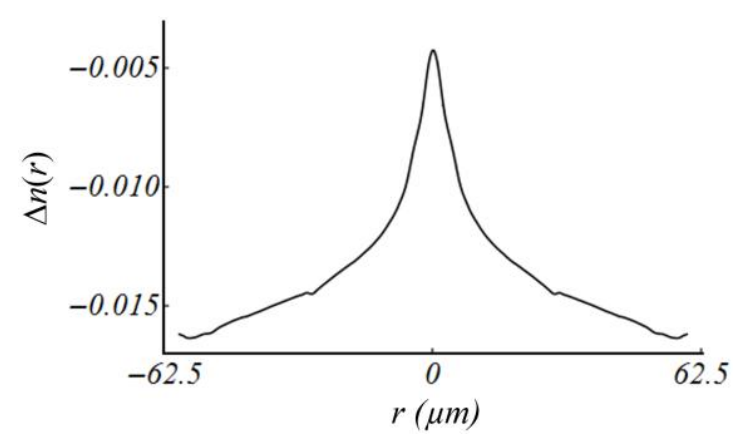

(a)

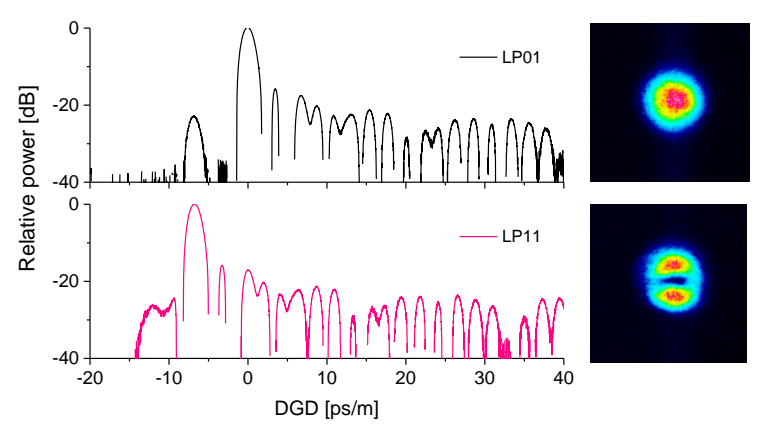

(b)

Fig. 1: (a) Measured refractive index profile (relative to undoped fused silica) of logarithmic (LOG) fibre [5] and (b) time-of-flight measurement for pure $\mathrm{LP}_{01}$ and $\mathrm{LP}_{11}$ mode excitation. 
between the two spatial modes is about -6.7 $\mathrm{ps} / \mathrm{m}$. Note that this is quite a large negative value, which means that the $L P_{11}$ mode is travelling much faster than $\mathrm{LP}_{01}$ mode. High modal purity of $>20 \mathrm{~dB}$ is readily achieved in this fibre and the guided modal intensity profiles are confirmed by a CCD camera (under $1550 \mathrm{~nm}$ CW laser excitation).

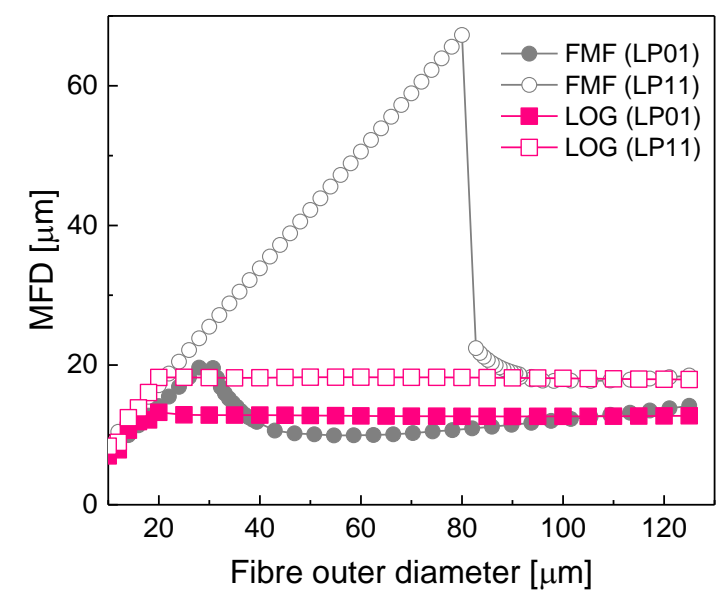

(a)
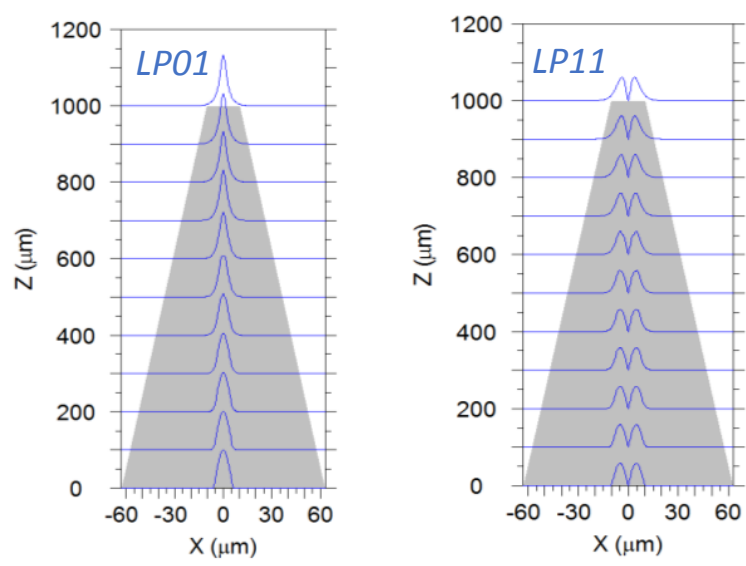

(b)

Fig. 2: (a) Change in MFDs of step-index FMF and LOG fibre along the fibre taper and (b) mode field evolution of two transverse modes in a LOG fibre.

In order to analyze the adiabatic criterion of the LOG fibres compared to conventional step-index FMFs, the MFDs of the fibres were calculated as a function of the outer diameter (OD) of the fibre taper. As shown in Fig. 2(a) going from right to left, i.e. down a taper transition, the MFDs of both spatial modes initially decrease slightly for stepindex FMFs but soon increase noticeably as the light guidance of the core becomes weaker and is gradually taken over by guidance at the cladding/air boundary. More importantly, the LP $\mathrm{P}_{11}$ modes cease to be guided along the core while the outer diameter is relatively large around 80 $\mu m$ (i.e. the HOMs experience a cutoff) and the MFD increases sharply. This sudden big MFD change of the HOMs is the primary reason that it is difficult to achieve an adiabatic taper transition from conventional step-index (or graded-index) FMFs. However, the MFD curve of the LOG fibre is nearly flat for both spatial modes until the fibre outer diameter becomes comparable to the MFD. This unique characteristics of constant MFDs along the taper makes LOG fibre a good candidate for the fabrication of HOM microfibres with negligible insertion loss. Figure 2(b) shows the calculated mode field evolution in a tapered LOG fibre. The beam sizes of both modes show only modest changes along the taper region even though only a very short taper transition $(\sim 1 \mathrm{~mm})$ is employed in this particular beam propagation simulation.

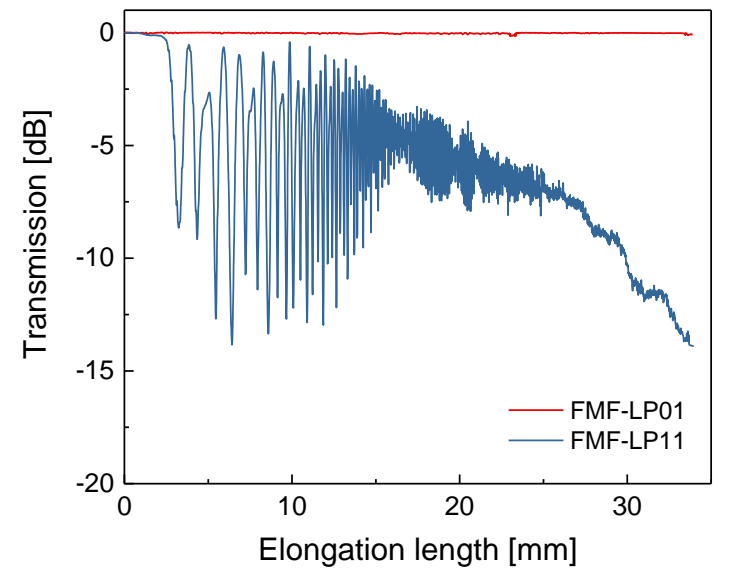

(a)

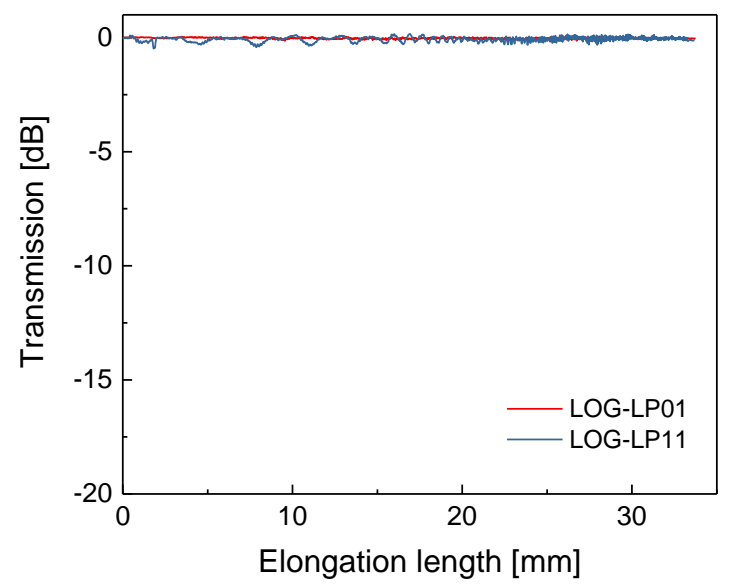

(b)

Fig. 3: Transmitted power evolution of both (a) step-index FMF and (b) LOG fibre for pure $\mathrm{LP}_{01}$ and $L P_{11}$ mode excitations as a function of pull length. 


\section{Microfibre fabrication}

By using the well-established flame brushing technique [6,7], both optical fibres were gradually tapered from $125 \mu \mathrm{m}$ to $2 \mu \mathrm{m}$ with an exponential taper profile (uniform waist length $=4 \mathrm{~mm}$, transition length $=15 \mathrm{~mm}$ ) and the transmitted optical power was monitored as a function of the pull length. As shown in Fig. 3(a), the $\mathrm{LP}_{11}$ mode of the step-index FMF experiences oscillatory power fluctuations due to the non-adiabatic taper transition during the tapering process, which is a signature of energy transfer from the core mode to the unwanted higher-order cladding modes, and as such it continuously loses some fraction of optical power, resulting in a higher insertion loss $(13.9 \mathrm{~dB})$. However, as expected, the $\mathrm{LP}_{11}$ mode of the LOG fibre in Fig. 3(b) shows no signature of power oscillation (i.e. adiabatic transition) and the total insertion loss of the $\mathrm{LP}_{11}$ mode was less than $0.11 \mathrm{~dB}$, comparable to that of $L P_{01}(0.03 \mathrm{~dB})$ in this experiment.

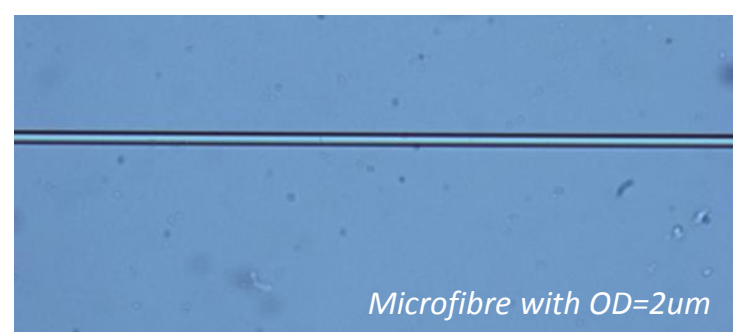

Fig. 4: Microscope image of the fabricated microfibre with an outer diameter of $2 \mu \mathrm{m}$.

The microscope image of the fabricated microfibre is shown in Fig. 4. The outer diameter of the taper waist was $\sim 2 \mu \mathrm{m}$ and the fabricated microfibre was protected/packaged with a commercially available fused fibre coupler packaging components (e.g. U-shaped glass substrate tube and outer stainless steel housing).

\section{Conclusion}

In conclusion, we have successfully fabricated low loss microfibres supporting higher order spatial modes using a logarithmic index fibre. This specialty optical fibre can provide an invariant mode field diameter along the fibre taper for all spatial modes and enable a very smooth mode transition from core modes to cladding modes (i.e. adiabatic taper transition). We have focused on the characterization of the two lowest order transverse modes $\left(\mathrm{LP}_{01}\right.$ and $\left.\mathrm{LP}_{11}\right)$ of this fibre and their microfibres were readily fabricated with very low insertion loss (typically less than 0.2 $\mathrm{dB}$ for both spatial modes). We believe that the low-loss HOM microfibres could prove a very useful tool for particle propulsion, microresonators and fan-in/fan-out device fabrication for space division multiplexed transmission applications.

\section{Acknowledgements}

This work was supported by the EPSRC funded "AirGuide Photonics" Programme Grant (EP/P030181/1), the STFC (ST/N000544/1) and the EC (Horizon 2020 contract 730890 "OPTICON").

\section{References}

[1] L. Tong et al., "Subwavelength diameter silica wires for low-loss optical wave guiding," Nature 426, 816 (2003).

[2] G. Brambilla et al., "Optical fiber nanowires and microwires: fabrication and applications," Adv. Opt. Photon. 1, 107 (2009).

[3] A. Maimaiti et al., "Higher order microfibre modes for dielectric particle trapping and propulsion," Sci. Rep. 5, 9077 (2015).

[4] R. Ravets et al., "A low-loss photonic silica nanofiber for higher-order modes," Opt. Express 21, 18325-18335 (2013).

[5] K. Harrington et al., "Endlessly adiabatic fiber with a logarithmic refractive index distribution," Optica 4, 1526 (2017).

[6] Y. Jung et al., "Broadband single-mode operation of standard optical fibers by using a sub-wavelength optical wire filter," Opt. Express 16, 14661 (2008).

[7] Y. Jung et al., "Optical microfiber coupler for broadband single mode operation," Opt. Express 17, 5273(2009). 